\title{
Extended Delivery Time Analysis of Cognitive Data Transmission over Multiple Primary Channels
}

\author{
Muhammad N. Khalid*, Muneer Usman*, Hong-Chuan Yang*, Mohamed-Slim Alouini* \\ *** Dept. of Electrical and Computer Engineering, University of Victoria, Victoria, Canada \\ ${ }^{*}$ Google Inc., Mountain View, USA \\ *Dept. Computer, Electrical and Mathematical Science and Engineering (CEMSE) Division, \\ King Abdullah University of Science and Technology (KAUST), Thuwal, Saudi Arabia \\ *mnkhalid@uvic.ca, *muneer.usman@gmail.com, ${ }^{*}$ hy@uvic.ca, ${ }^{*}$ slim.alouini@kaust.edu.sa
}

\begin{abstract}
Cognitive radio (CR) systems can improve radio spectrum utilization by allowing secondary access of underutilized spectrum resources. With interweave cognitive implementation, secondary users have to wait for spectrum opportunities before their transmission. Therefore, the total delivery time of secondary transmission consists of both waiting slots and transmission slots. In this work, we study the resulting extended delivery time (EDT) of secondary transmission for a fixed amount of data over multiple primary channels. In particular, a birthdeath process is introduced to model the number of available primary channels, based on which, we derive the distribution function of the EDT for both continuous and periodic sensing cases. We also present selected numerical results to illustrate the mathematical formulation.
\end{abstract}

Index Terms-Cognitive radio, birth-death process, Markov chains, extended delivery time.

\section{INTRODUCTION}

Cognitive radio improves spectrum utilization by allowing unlicensed secondary users (SU) to access the under-utilized licensed frequency bands opportunistically without significantly affecting the performance of the licensed primary users (PU) [1]. With interweave implementation, SU need to locate spectrum holes (i.e. unoccupied licensed frequency bands of the primary system) through spectrum sensing. Specifically, SU can only access the channel that is not being used by PU and must stop its transmission as soon as PU reappear. One of the main challenge of this implementation is spectrum sensing. In order to return the channel to $\mathrm{PU}$ and then to re-access the same or another channel to complete the transmission, spectrum handoff techniques are also required. The SU transmission of a given amount of data may involve multiple spectrum handoffs, which will result in extra transmission delay. The total time needed for data transmission will consist of all the waiting periods before accessing the primary channels and the time needed for completing the transmission, which is called the extended delivery time (EDT) [2].

Delay and throughput analysis for secondary systems have been a constant focus for cognitive systems. [2] derived analytical bounds on the throughput and transmission delay of secondary transmission in cognitive radio network. For interweave transmission, [3] investigates the average service and waiting time for $\mathrm{SU}$ in a single transmission slot for the general primary traffic model. An analytical expression for the probability density function of the service time available to a secondary user during a fixed time slot was derived in [4]. A lower bound on the service time of SU for packets with length varying according to some general random distribution was derived in [5]. [6] calculates the effect of multiple spectrum handoffs on the average EDT of a secondary packet in a cognitive radio network with multiple channels and users. Probability of successful transmission in cooperative wireless communication with the hard delay constraint was studied in [7].

When the SU transmission is interrupted due to the reappearance of PU, secondary system can adopt either the work preserving strategy or non-work preserving strategy. With the work preserving strategy, the SU transmission can continue from the point it was interrupted [6], whereas, in non-work preserving strategy, the interrupted secondary transmission must restart from the beginning and therefore previous transmission is wasted [2]. The work preserving strategy can be achieved with the use of rateless codes, e.g. Fountain code [11] and Raptor code [12]. This strategy also applies to small and individually coded sub-packet transmission scenarios. [13], [14] derived the exact expressions of the probability density function (PDF) for the EDT of SU transmission over a single PU channel and further used these expressions to analyze the average delay performance of secondary transmission. As far as we know, [13], [14] are the only previous work where exact expressions for the PDF of EDT are derived. However, these expressions are limited to one PU channel scenario.

In this paper, we investigate the delay performance of secondary transmission over multiple PU channels with work preserving strategy under the interweave implementation. We introduce a birth-death process based approach to analyze EDT for the SU transmission over multiple primary channels and derive analytical expression of PDF and probability mass function (PMF) for the EDT of a fixed amount of secondary data. The accuracy of this approach is later verified through numerical simulations. To the best of our knowledge, the use of birth-death process to model a secondary user transmission over multiple primary channels is a completely new concept. Through selected numerical examples, we show that interweave transmission over multiple primary channels can effectively reduce the EDT. 


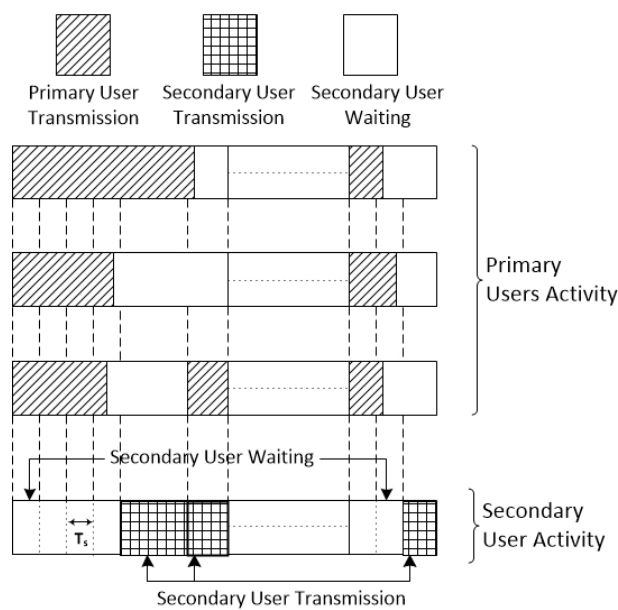

Fig. 1. Illustration of secondary access of multiple PU channels with periodic sensing.

The rest of the paper is organized as follows. Section II presents the system model and problem formulation. This section also introduces the birth-death process and its reduction to a two-state continuous time Markov chain. In Section III, we use the intermediate results from Section II to find the distribution functions of EDT for both continuous and periodic sensing cases. Selected numerical examples, verifying the analytical results from Section III, are presented in section IV. Finally, Section V presents some concluding remarks.

\section{System Model ANd Problem Formulation}

We consider a cognitive transmission scenario where a secondary transmitter and receiver pair opportunistically accesses $N$ channels of a primary system. The occupancy of these channels by PU varies independently according to a homogeneous continuous time Markov chain with average busy duration of $\lambda$ and average free duration of $\mu$. Thus, the duration of busy and free time slots on each PU channel are independently exponential distributed. SU adopts an interweave implementation strategy. To be more precise, SU can only access a channel not used by PU and stop the access of that channel when PU starts transmission. Thus, no interference is caused to PU transmission. If a PU channel becomes unavailable, SU will treat it as a busy channel and try to access other channels instead.

SU monitors the activity of PU through spectrum sensing. Specific spectrum sensing techniques have been discussed in details in [15] and [16]. We assume perfect sensing for analytical tractability. The case of imperfect spectrum sensing will be considered in future work. To ensure no interference with the PU activity, we assume that during transmission, SU constantly monitors the PU activity of that particular channel. When the PU reappears during the SU transmission, SU will either switch to another free channel and continue transmission or wait for a free channel before transmission.
Meanwhile, when not transmitting, SU can monitor the activity of PU channels using two different approaches, i.e. continuous and periodic sensing. With continuous sensing, SU continuously senses all the channels and start their data transmission as soon as a free channel is found, whereas, in periodic sensing case, SU senses the primary channels periodically, with a sensing period of $T_{s}$. We assume that the duration of spectrum sensing is very short and negligible compared to transmission duration.

The continuous period of time during which SU transmits over a particular channel is referred to as a transmission slot. Similarly, continuous period of time, during which SU is not transmitting, is referred to as a waiting slot. With periodic sensing, the waiting slot may also include the time period when a PU channel has become free but SU has not yet sensed the channels. Fig. 1 illustrates the effect of PU activity on transmission and waiting slots for periodic sensing case.

\section{A. Birth-Death Process}

In this work, we analyze the total data delivery time (which consists of a interleaved sequence of transmission slots and waiting slots) of a secondary system over multiple primary channels. The total delivery time is given by $T_{E D}=T_{t r}+T_{w}$, where $T_{t r}$ is the data transmission time and $T_{w}$ is total waiting time of SU. In general both $T_{t r}$ and $T_{w}$ are some random variables, where $T_{w}$ depends on $T_{t r}$, the PU behavior and sensing strategies, and $T_{t r}$ depends upon the channel conditions and the $\mathrm{SU}$ data size. Here, we assume $T_{t r}$ is constant. As such, to derive the exact distribution of EDT, we need to derive the exact distribution function of $T_{w}$ [14].

We introduce a birth-death process to model the variation of the number of free primary channels. An illustration of this birth-death process is shown in Fig. 2, where state $i$ corresponds $i$ PU channels are free and the remaining $N-i$ ones are busy. Here, a birth refers to an additional primary channel going from busy state to free state. Similarly, a death corresponds to the event that a free primary channel becomes busy. Since the duration of each primary channel in free state is exponential with rate $\frac{1}{\mu}$, the sojourn time for state $i$ is the minimum of $i$ i.i.d exponential random variables, which is also exponential [17] with rate $\frac{1}{\mu_{i}}=\frac{i}{\mu}, i=0,1,2, \ldots, N$. Similarly, the birth rate from state $i$ is $\frac{1}{\lambda_{i}}=\frac{N-i}{\lambda}$.

\section{B. Two-State Markov Chain from the Birth-Death Process}

Now, by observing the transmission pattern in Fig. 1, it becomes clear that the SU will only be in the waiting slot when the system is in state 0 . We consolidate the complete multi-state birth death process into a two state continuoustime Markov chain shown in Fig. 3 where $\frac{1}{\lambda_{0}}=\frac{N}{\lambda}$ is rate of leaving state 0 , and $\frac{1}{\mu^{*}}$ is the rate of coming back to state 0 . Assuming at time $t=0$, the process goes from state 0 to state 1 , and $T_{1}$ represents the time it takes for the process to go back to state 0 . Then, we have

$$
\mu^{*}=E\left[T_{1}\right] .
$$




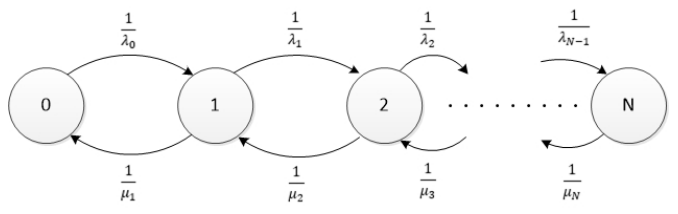

Fig. 2. Illustration of finite state birth-death process for the number of free primary channels from a total of $N$ primary channels.

Now let $T_{i}$ denote the time during that it takes for the process starting from state $i$, to enter state $i-1$, for $1 \leq i \leq N$. Then $E\left[T_{i}\right], i \leq N$, can be obtained (see Appendix A) as

$$
E\left[T_{i}\right]=\mu(i-1) !\left[\sum_{n=0}^{N-i} \frac{(N-i) !}{n !}\left(\frac{\mu}{\lambda}\right)^{N-i-n} \frac{1}{(N-n) !}\right] .
$$

In particular, $E\left[T_{1}\right]$ is the average time duration that takes for the number of free primary channels to go back zero is given by

$$
\mu^{*}=E\left[T_{1}\right]=\mu\left[\sum_{n=0}^{N-1} \frac{(N-1) !}{n !}\left(\frac{\mu}{\lambda}\right)^{N-1-n} \frac{1}{(N-n) !}\right]
$$

\section{EXtended Delivery Time ANAlysis}

We can apply this reduced two-state continuous time Markov chain to analyze the EDT for the SU based on the results in [13].

\section{A. Continuous Sensing}

For continuous sensing case, the PDF of EDT $f_{T_{E D}}(t)$ for single PU channel case was given by [13]

$$
\begin{gathered}
f_{T_{E D}}(t)=u\left(t-T_{t r}\right) \frac{1}{\lambda+\mu} e^{\frac{-\left(t-T_{t r}\right)}{\lambda}} e^{\frac{-T_{t r}}{\mu}} \\
\times\left[I_{0}\left(2 \sqrt{\frac{T_{t r}\left(t-T_{t r}\right)}{\mu \lambda}}\right)+\sqrt{\frac{T_{t r} \mu}{\lambda\left(t-T_{t r}\right)}}\right. \\
\left.\times I_{1}\left(2 \sqrt{\frac{T_{t r}\left(t-T_{t r}\right)}{\lambda \mu}}\right)\right]+\frac{\mu}{\lambda+\mu} e^{\frac{-T_{t r}}{\mu}} \delta\left(t-T_{t r}\right),
\end{gathered}
$$

where $u(\cdot)$ is unit step function, $I_{n}(\cdot)$ is the modified Bessel function of the first kind of order $n, \lambda$ is the average busy time for the primary user, $\mu$ is the average free time for the primary user and $\delta(t)$ is the delta function.

Now, as seen in Fig. 3, the average free time for our reduced two-state continuous markov chain is $\mu^{*}$ and the average busy time is $\lambda_{0}=\frac{\lambda}{N}$. So, by replacing $\mu$ and $\lambda$ in Eq.(4) with

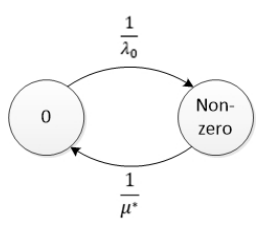

Fig. 3. Illustration of the condensed two state continuous time Markov chain

$\mu^{*}$ and $\frac{\lambda}{N}$, respectively, the PDF of the EDT $T_{E D}$ for the continuous sensing case over $N$ primary channels is given by

$$
\begin{gathered}
f_{T_{E D}}(t)=u\left(t-T_{t r}\right) \frac{N}{\lambda+N \mu^{*}} e^{\frac{-N\left(t-T_{t r}\right)}{\lambda}} e^{\frac{-T_{t r}}{\mu^{*}}} \\
\times\left[I_{0}\left(2 \sqrt{\frac{N T_{t r}\left(t-T_{t r}\right)}{\mu^{*} \lambda}}\right)+\sqrt{\frac{N T_{t r} \mu^{*}}{\lambda\left(t-T_{t r}\right)}}\right. \\
\left.\times I_{1}\left(2 \sqrt{\frac{N T_{t r}\left(t-T_{t r}\right)}{\mu^{*} \lambda}}\right)\right]+\frac{N \mu^{*}}{\lambda+N \mu^{*}} e^{\frac{-T_{t r}}{\mu^{*}}} \delta\left(t-T_{t r}\right) .
\end{gathered}
$$

\section{B. Periodic Sensing}

The PMF of EDT of one PU for periodic sensing case from [13] is given by

$$
\begin{aligned}
& \operatorname{Pr}\left[T_{E D}=\right.\left.n T_{s}+T_{t r}\right]=\frac{\lambda}{\lambda+\mu}(1-\beta) \beta^{n-1} e^{\frac{-T_{t r}}{\mu}} \\
& \times{ }_{1} F_{1}\left(1-n ; 1 ; \frac{-T_{t r}(1-\beta)}{\mu \beta}\right) u[n] \\
&+\frac{\mu}{\lambda+\mu}\left[\left(\frac{T_{t r}(1-\beta) \beta^{n-1}}{\mu}\right) e^{\frac{-T_{t r}}{\mu}}\right. \\
&\left.\times{ }_{1} F_{1}\left(1-n ; 2 ; \frac{-T_{t r}(1-\beta)}{\mu \beta}\right) u[n-1]\right] \\
&+\frac{\mu}{\lambda+\mu} e^{\frac{-T_{t r}}{\mu}} \delta[n],
\end{aligned}
$$

where $n$ is total number of sensing instances, ${ }_{1} F_{1}(., .,$.$) is the$ generalized Hyper-geometric function and $\beta$ is the probability that the primary user is on at the sensing instance given it was sensed free $T_{s}$ time period earlier and given by

$$
\beta=\frac{\lambda}{\lambda+\mu}+\frac{\mu}{\lambda+\mu} e^{-\left(\frac{1}{\lambda}+\frac{1}{\mu}\right) T_{s}} .
$$

Now, similar to the continuous sensing case, substituting $\mu$ and $\lambda$ from Eqs. (6) and (7) by $\mu^{*}$ and $\frac{\lambda}{N}$ respectively, we get

$$
\beta_{1}=\frac{\lambda}{\lambda+N \mu^{*}}+\frac{N \mu^{*}}{\lambda+N \mu^{*}} e^{-\left(\frac{N}{\lambda}+\frac{1}{\mu^{*}}\right) T_{s}},
$$




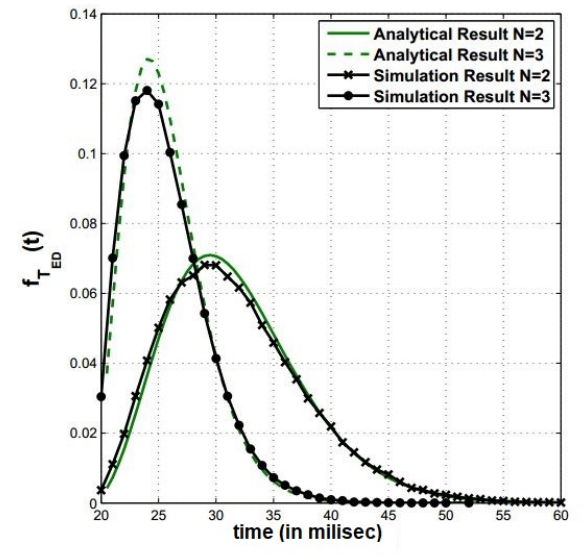

Fig. 4. Simulation verification of the analytical PDF of $T_{E D}$ with continuous sensing $\left(T_{t r}=20 \mathrm{~ms}, \lambda=3\right.$ and $\mu=2$.)

and the PMF of the EDT $T_{E D}$ for the periodic sensing case over $N$ primary channels is given by

$$
\begin{aligned}
& \operatorname{Pr}\left[T_{E D}\right.\left.=n T_{s}+T_{t r}\right]=\frac{\lambda}{\lambda+N \mu^{*}}\left(1-\beta_{1}\right) \beta_{1}{ }^{n-1} e^{\frac{-T_{t r}}{\mu^{*}}} \\
& \times{ }_{1} F_{1}\left(1-n ; 1 ; \frac{-T_{t r}\left(1-\beta_{1}\right)}{\mu^{*} \beta_{1}}\right) u[n] \\
&+ \frac{N \mu^{*}}{\lambda+N \mu^{*}}\left[\left(\frac{T_{t r}\left(1-\beta_{1}\right) \beta_{1}{ }^{n-1}}{\mu^{*}}\right) e^{\frac{-T_{t r}}{\mu^{*}}}\right. \\
& \times\left.{ }_{1} F_{1}\left(1-n ; 2 ; \frac{-T_{t r}\left(1-\beta_{1}\right)}{\mu^{*} \beta_{1}}\right) u[n-1]\right] \\
&+\frac{N \mu^{*}}{\lambda+N \mu^{*}} e^{\frac{-T_{t r}}{\mu^{*}}} \delta[n] .
\end{aligned}
$$

\section{NUMERICAL EXAMPLES}

Fig. 4 plots the analytical result for the PDF of EDT for the continuous sensing scenario, given in Eq.(5). The corresponding plots for the simulation results for the different values of $N$, are also shown. The near perfect match between the analytical and simulation results verify our birth-death process based analytical approach ${ }^{1}$. Also, the general trend of decreasing average EDT with an increase in the number of primary channels $N$ also gets verified. Fig. 5 plots the PMF of EDT for periodic sensing case for different number of primary channels $N$. The corresponding simulation results are also shown. The plot shows that the analytical results reconcile with the simulation results very well. Also, these plots verify the expected trend of decreasing in average EDT for periodic sensing as the number of primary channels $N$ increases. Fig. 6 shows the analytical PMF envelope of the EDT with the

\footnotetext{
${ }^{1}$ The slight mismatch may come from the uncertainty of the initial state of the birth-death process when the data transmission starts. Note that our analysis assumes that the process always starts from a single free PU channel.
}

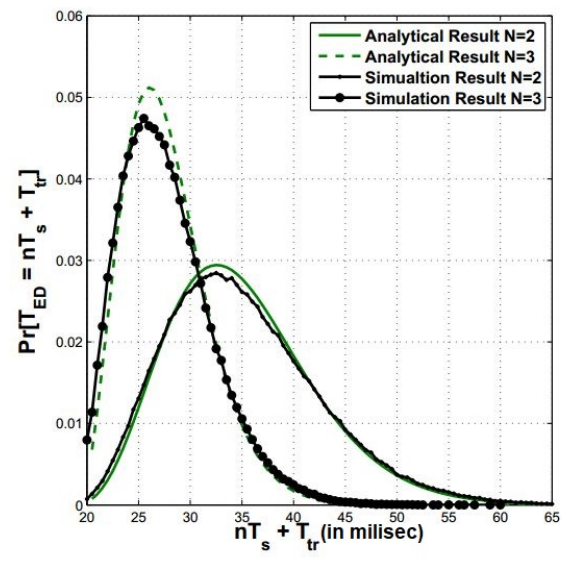

Fig. 5. Simulation verification of the analytical PMF of $T_{E D}$ with periodic sensing $\left(T_{t r}=20 \mathrm{~ms}, \lambda=3, \mu=2\right.$ and $\left.T_{s}=0.5 \mathrm{~s}\right)$.

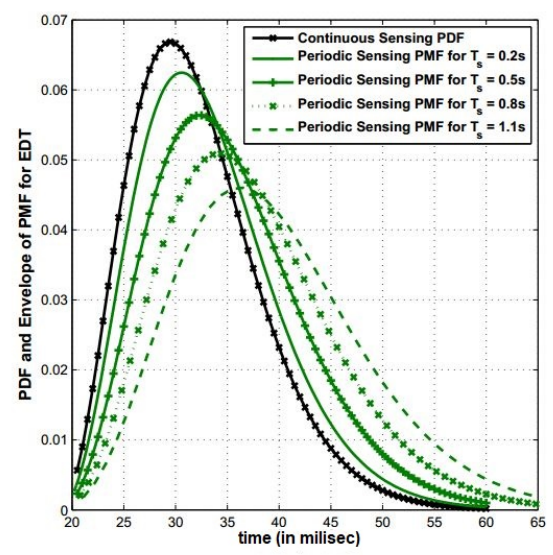

Fig. 6. Analytical distribution of EDT with the continuous and periodic sensing $\left(N=3, T_{t r}=10 \mathrm{~ms}, \lambda=5\right.$ and $\left.\mu=2\right)$.

periodic sensing case corresponding to the different sensing periods $T_{s}$ along with the analytical PDF of the continuous sensing case. As $T_{s}$ approaches 0 , the performance of periodic sensing converges to that of continuous sensing case, which is as expected.

\section{CONCLUSION}

This paper studied the extended delivery time of secondary data transmission over multiple primary user channels in an interweave cognitive setup. A birth-death process is introduced to model the secondary transmission. The probability density function and probability mass function of EDT for a fixed amount of data were derived for continuous sensing and 
periodic sensing cases, respectively. Simulation results were presented to verify the analytical results. Near perfect match between the analytical and the simulation results verified that a birth-death process can be used to model and analyze secondary transmission over multiple primary channels. Ongoing effort are considering the effect of non-identical PU behaviors and imperfect spectrum sensing.

\section{APPENDiX A}

In this appendix, we derive the expression for $E\left[T_{i}\right]$, which denotes the average time that it takes for the process, starting from state $i$, to enter state $i-1$, for $1 \leq i \leq N$. We will recursively compute $E\left[T_{i}\right], i \leq N$, by starting from $i=N$. Since $T_{N}$ is exponential with rate $\frac{1}{\mu_{N}}$, we have

$$
E\left[T_{N}\right]=\mu_{N}=\frac{\mu}{N} .
$$

For $1 \leq i \leq N$, we condition on whether the first transition is a birth or a death. That is, let

$I_{i}= \begin{cases}1, & \text { if the first transition from } i \text { is to } i-1(\text { is death }) \\ 0, & \text { if the first transition from } i \text { is to } i+1(\text { is birth })\end{cases}$ and note that

$$
E\left[T_{i} \mid \text { death }\right]=E\left[T_{i} \mid I_{i}=1\right]=\frac{1}{\frac{1}{\lambda_{i}}+\frac{1}{\mu_{i}}},
$$

and

$E\left[T_{i} \mid\right.$ birth $]=E\left[T_{i} \mid I_{i}=0\right]=\frac{1}{\frac{1}{\lambda_{i}}+\frac{1}{\mu_{i}}}+E\left[T_{i+1}\right]+E\left[T_{i}\right]$.

This follows, since independent of whether the first transition is either a birth or a death, the time for this transition is exponential with rate $\frac{1}{\lambda_{i}}+\frac{1}{\mu_{i}}$. If this first transition is a death, then the number of free primary channels becomes $i-1$. Therefore, no need for any additional time. If it is a birth, then the number of free primary channels becomes $i+1$ and then the additional time needed to reach $i-1$ is equal to the time that it takes to return to $i$ (this is $E\left[T_{i+1}\right]$ ) plus the time that it takes to reach $i-1$ (this is $E\left[T_{i}\right]$ ). Now, as the probability that the first transition is a death is $\frac{\frac{1}{\mu_{i}}}{\frac{1}{\lambda_{i}}+\frac{1}{\mu_{i}}}$, using Eqs. (11) and (12) we get

$$
E\left[T_{i}\right]=\frac{1}{\frac{1}{\lambda_{i}}+\frac{1}{\mu_{i}}}+\frac{\frac{1}{\lambda_{i}}}{\frac{1}{\lambda_{i}}+\frac{1}{\mu_{i}}}\left(E\left[T_{i}\right]+E\left[T_{i+1}\right]\right),
$$

or, equivalently,

$$
E\left[T_{i}\right]=\mu_{i}+\frac{\mu_{i}}{\lambda_{i}} E\left[T_{i+1}\right] .
$$

Starting from $E\left[T_{N}\right]=\frac{\mu}{N}$, Eq. (14) provides an efficient method to successively compute $E\left[T_{N-1}\right], E\left[T_{N-2}\right]$, and up to $E\left[T_{1}\right]$. So, starting from $E\left[T_{N}\right]$ and using Eq. (14), we see that

$$
E\left[T_{N-1}\right]=\mu(N-2) !\left[\frac{1}{(N-1) !}+\left(\frac{\mu}{\lambda}\right) \frac{1}{N !}\right],
$$

$$
\begin{aligned}
E\left[T_{N-2}\right] & =\mu(N-3) !\left[\frac{1}{(N-2) !}\right. \\
& \left.+2\left(\frac{\mu}{\lambda}\right) \frac{1}{(N-1) !}+2 \cdot 1\left(\frac{\mu}{\lambda}\right)^{2} \frac{1}{N !}\right],
\end{aligned}
$$

and, in general,

$$
E\left[T_{i}\right]=\mu(i-1) !\left[\sum_{n=0}^{N-i} \frac{(N-i) !}{n !}\left(\frac{\mu}{\lambda}\right)^{N-i-n} \frac{1}{(N-n) !}\right] .
$$

\section{REFERENCES}

[1] "Report of the spectrum efficiency working group," Federal Communications Commission Spectrum Policy Task Force, Tech. Rep., Nov. 2002. Download available at http://transition.fcc.gov/sptf/files/SEWGFinalReport1.pdf

[2] F. Borgonovo, M. Cesana, and L. Fratta, "Throughput and delay bounds for cognitive transmissions, in Advances in Ad Hoc Networking ( $P$. Cuenca, C. Guerrero, R. Puigjaner, and B. Serra, eds.), vol. 265 of IFIP International Federation for Information Processing, pp. 179-190, Springer US, 2008.

[3] F. Gaaloul, H.-C. Yang, R. Radaydeh, and M.-S. Alouini, "Switch based opportunistic spectrum access for general primary user traffic model," IEEE Wireless Commun. Lett., vol. 1, pp. 424-427, October 2012.

[4] Z. Liang and D. Zhao, "Quality of service performance of a cognitive radio sensor network," in Proc. IEEE Int. Conf. Commun. (ICC), 2010 , pp. 1-5, May 2010.

[5] M. Luis, R. Oliveria, R. Dinis and L. Bernardo, "Characterization of Opportunistic Service Time in Cognitive Radio Networks," IEEE trans. on Cognitive communications and Networking, vol. 2, no.3, pp. 288300, September 2016.

[6] C.-W. Wang and L.-C. Wang, "Analysis of reactive spectrum handoff in cognitive radio networks," IEEE J. Sel. Areas Commun., vol. 30, pp. 2016-2028, November 2012.

[7] S. Kandeepan, C. Saradhi, M. Filo, and R. Piesiewicz, "Delay analysis of cooperative communication with opportunistic relay access," in Proc. IEEE 73rd Veh. Technol. Conf. (VTC Spring), 2011, pp. 1-5, May 2011.

[8] F. Khan, K. Tourki, M.-S. Alouini, and K. Qaraqe, "Delay performance of a broadcast spectrum sharing network in Nakagami-m fading," IEEE Trans. Veh. Technol., vol. 63, pp. 1350-1364, March 2014.

[9] L. Sibomana, H.-J. Zepernick, H. Tran, and C. Kabiri, "Packet transmission time for cognitive radio networks considering interference from primary user," in 9th Int. Wireless Commun. and Mobile Computing Conf. (IWCMC), 2013, pp. 791-796, July 2013.

[10] L. Musavian and S. Aissa, "Effective capacity of delay-constrained cognitive radio in nakagami fading channels," IEEE Trans. Wireless Commun., vol. 9, pp. 1054-1062, March 2010.

[11] D. J. C. MacKay, "Fountain codes," IEEE Proc.-Commun., vol. 152, pp.1062-1068, Dec 2005.

[12] J. Castura and Y. Mao, "Rateless coding over fading channels," IEEE Commun. Lett., vol. 10, pp. 46-48, Jan 2006.

[13] M. Usman, H.-C. Yang, M.-S. Alouni, "Extended Delivery Time Analysis for Cognitive Packet Transmission with Application to Secondary Queuing Analysis," IEEE Trans. on Wireless Communications, vol. 14, pp. 5300 - 5312, Oct. 2015.

[14] M. Usman, H.-C. Yang, M.-S. Alouni, "Further Results on Extended Delivery Time for Secondary Packet Transmission," IEEE Trans. on Wireless Communications, vol. 16, issue 10, pp. 6451-6459, Oct. 2017.

[15] I. F. Akyildiz, W.-Y. Lee, M. C. Vuran, and S. Mohanty, "Next generation/dynamic spectrum access/cognitive radio wireless networks: A survey," Computer Networks J., vol. 50, no. 13, pp. 2127 - 2159, Sep. 2006.

[16] M. Islam, C. Koh, S. W. Oh, X. Qing, Y. Lai, C. Wang, Y.-C. Liang, B. Toh, F. Chin, G. Tan, and W. Toh, "Spectrum survey in Singapore: Occupancy measurements and analyses," in Proc. 3rd Int. Conf. Cognitive Radio Oriented Wireless Netw. and Commun., pp. 1-7, May 2008.

[17] "The Exponential Distribution and the Poission Process," in Introduction to Probability Models, pp. 302-317, Elsevier US, 2010. 